\section{Formación de microfisuras dentinarias en la preparacion químico mecánica del conducto radicular con los sistemas protaper universal, protaper next y twisted file adaptive.}

\author{
Dentinal microcracks formation in the root canal in chemical \\ mechanical preparation with protaper universal, protaper next and \\ twisted file adaptive
}

\begin{abstract}
Resumen
Objetivo :La presente investigación buscó evaluar la formación de microfisuras dentinarias en el conducto radicular después de la preparación químico mecánica con los sistemas Protaper Universal, Protaper Next y TF Adaptive. Para luego poder determinar cual de los sistemas genera menor número de microfisuras luego de la preparación quimico mecanica. Materiales y método: Se seleccionaron 38 premolares mandibulares recién extraidos, que tuvieran un solo conducto radicular.

La muestra se dividio en tres grupos experimentales $(n=11)$ correspondiente a cada sistema de preparación químico mecánica. Grupo A: Protaper Universal, Grupo B: Protaper Next, Grupo C: TF Adaptive. Y un cuarto grupo $(n=5)$ que correspondía al grupo de control negativo. Luego de la preparación químico mecánica se realizaron cortes horizontales a $4 \mathrm{~mm}, 8 \mathrm{~mm}$ y $12 \mathrm{~mm}$ desde el ápice, los cuales fueron evaluados con un estereomicroscopio de 20x donde se determinó la presencia de microfisuras. Resultados: El Protaper Universal, produce microfisuras en comparación al Protaper Next y el TF Adaptive siendo esta diferencia estadisticamente significativa $(\mathrm{P}<.05)$. A los $12 \mathrm{~mm}$ el sistema TF Adaptive presenta mayor número de microfisuras sin ser esta estadisticamente significativa. Conclusión: Los sistemas Protaper Next y Twisted File Adaptive tienden a generar menor cantidad de microfisuras dentinarias en comparación al sistema Protaper Universal en el tercio apical.
\end{abstract}

Palabras clave: Preparación del Conducto Radicular - Microfisuras - Instrumentos rotatorios.

\begin{abstract}
Object: evaluate the formation of microcracks in the dental root canal after chemical mechanical preparation with Protaper Universal, Protaper Next and TF Adaptive systems. Then to determine which systems generates fewer microcracks after chemical mechanical preparation. Materials and méthod: 38 freshly extracted mandibular premolars, which have a single root canal were selected. The sample was divided into three for each chemical mechanical preparation system experimental groups $(n=11)$. Group A: Protaper Universal Group B: Protaper Next, Group C: Adaptive TF. And a fourth group ( $\mathrm{n}=$ 5) corresponding to the negative control group. After chemical mechanical preparation horizontal slices $4 \mathrm{~mm}, 8 \mathrm{~mm}$ and $12 \mathrm{~mm}$ from the apex, which were evaluated with a 20x stereomicroscope where the presence of microcracks was determined were performed. Results: The Protaper Universival produces microcracks compared to Protaper Next and Adaptive TF with a statistically significant difference $(\mathrm{P}$ $<.05)$. At $12 \mathrm{~mm}$ the Adaptive TF system has more micro cracks without being this statistically significant. Conclusions: The Next Protaper and Twisted File Adaptive systems tend to generate fewer microcracks dentin compared to Protaper Universal system in the apical third.
\end{abstract} Keywords: Root Canal Preparations - Microcracks - Root - Rotary files.
Artículo Original

*Jara Castro ,Marisa, ${ }^{1}$ Peña Veliz Ines, ${ }^{2}$ Sequeiros Castillo,Maruxia, ${ }^{2}$ Salazar alfaro,Karina, ${ }^{2}$. Flores Pozo, Suellen. ${ }^{2}$

1. Departamento de Estomatologia Rehabiltadora UNMSM

2. Segunda Especialidad en Carielogia y Endodoncia de la UNMSM.

Correspondencia:

*Jara Castro, Marisa

Correo electrónico: marisareina@hotmail.com

Facultad de Odontología de la Universidad Nacional Mayor de San Marcos.

Av. Germán Amezaga 675. Lima 1, Perú

Coautores

Peńa Veliz, Ines

hines_pv@hotmail.com

Sequeiros Castillo, Maruxia

maruxiasc@gmail.com

Salazar Alfaro, Karina

ksalazar.denteik@gmail.com

Flores Pozo, Suellen

suellen_17@hotmail.com

Fecha de Recepcion : 11/07/2016

Fecha de Aceptación: 01/09/2016 


\section{Introducción}

La preparación del canal radicular es uno de los más importantes pasos en el éxito del tratamiento del conducto radicular, en la actualidad los sistemas rotatorios usan instrumentos de níqueltitanio, que podrían dar lugar a algunas complicaciones durante la preparación, tales como perforaciones ${ }^{1}$ traslape del conducto radicular y la formación de escalones ${ }^{2}$ separación de instrumentos (fracturas) ${ }^{3}$ y grietas o micro fisuras de la dentina ${ }^{4}$. La fractura radicular vertical, podría producirse como consecuencia de micro fisuras o grietas de la denti$\mathrm{na}^{5}$, pudiendo conducir a la extracción del diente ${ }^{6-9}$.

Actualmente la preparación mecanizada es uno de los avances dentro de los tratamientos endodónticos, por lo cual los fabricantes han tratado de mejorar los instrumentos (más fácil, mayor resistencia, más rápido y mejor conformación del conducto radicular), sin dejar de lado los principios de la preparación del conducto radicular. Esta investigación busca evaluar cuál de los sistemas: Protaper universal, Protaper Next y Twisted File Adaptive genera menor número de micro fisuras y grietas en el conducto radicular durante la conformación de los conductos radiculares.

\section{Materiales y método}

El tipo de Estudio fue Experimental, transversal, comparativo, prospectivo. El tamaño de muestra fueron 38 piezas permanentes premolares uniradiculares humanas, con criterios de exclusión fueron dientes con caries, con restauración previa raíces con curvatura mayor a $10^{\circ}$, y dientes con fisuras externas, las cuales fueron inspeccionadas con un estéreo microscopio 20x para corroborar que las piezas no presenten grietas externas. Se tomaron radiografías con dos tipos de angulaciones (vestibulolinguales y mesiodistales) para verificar que los dientes no tengan alguna fractura.

Se estandarizo la muestra cortando a 15 $\mathrm{mm}$ del ápice hacia coronal, con pieza de baja velocidad con refrigeración constante $\left(\mathrm{KAVO}^{\odot}\right)$.

La longitud del canal radicular se midió con una lima k \#10 hasta que la punta de la lima fue visible en el foramen apical. La distancia entre la punta de la lima y la referencia coronal se definió como la longitud del canal. La longitud de trabajo, se estableció restando $1 \mathrm{~mm}$ de esta longitud.

Los conductos radiculares serán irrigados con solución de hipoclorito de sodio $1 \%$ después de cada cambio de instrumento.

1. Grupo 1 (control): 5 piezas dentarias como control negativo, sin trabajar

2. Grupo 2: 11 piezas dentarias trabajadas con limas Protaper Universal. Se utilizaran con la secuencia de PROTAPER universal SX y luego $\mathrm{X} 1, \mathrm{X} 2, \mathrm{~F} 1, \mathrm{~F} 2, \mathrm{~F} 3$ con una velocidad de rotación de $300 \mathrm{rpm}$, con movimientos de cepillado.

3. Grupo 3: 11 piezas dentarias trabajadas con limas Protaper Next. $\mathrm{Se}$ utilizaron con la secuencia siguiente X1, X2, X3, X4 y X5 en una velocidad de rotación de 300 rpm, con movimientos de cepillado similar al usado en Protaper Universal.

4. Grupo 4: 11 piezas dentarias trabajadas con limas Twisted File Adaptative con movimiento continuo sin presión apical (SybronEndo, Glendora, CA).

La Preparación Químico-Mecánica de la muestra se realizó con tres tipos de sistemas rotatorios: Protaper Universal, Protaper Next y Twisted File Adaptive según las indicaciones de cada fabricante. Posterior a la preparación químico mecánica se realizaron cortes transversales a $4 \mathrm{~mm}, 8 \mathrm{~mm}$ y $12 \mathrm{~mm}$; las muestras fueron llevadas al estereomicroscopio LEICA E24 HD a 20x para la observación y registro de microfisuras dentinarias.

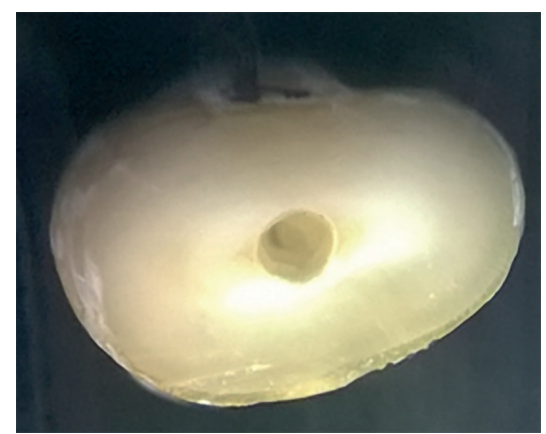

Figura $\mathrm{N}^{\circ}$ 1. Ausencia de Microfisura Dentinaria.

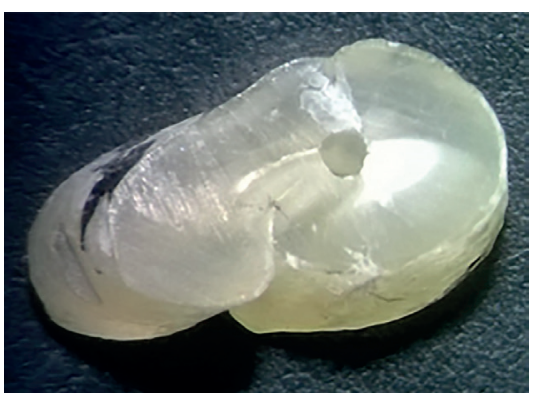

Figura $\mathrm{N}^{\circ} \mathbf{2}$. Presencia de Micro fisura Dentinaria.

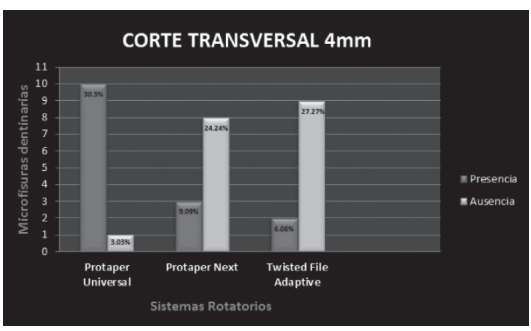

Figura $\mathrm{N}^{\circ}$ 3: Número y porcentaje de Microfisuras dentinarias a $4 \mathrm{~mm}$ después de la instrumentación con diferentes sistemas.

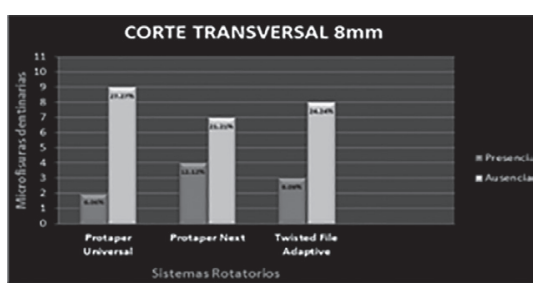

Figura $\mathrm{N}^{\circ}$ 3: Número y porcentaje de Microfisuras dentinarias a $8 \mathrm{~mm}$ después de la instrumentación con diferentes sistemas.

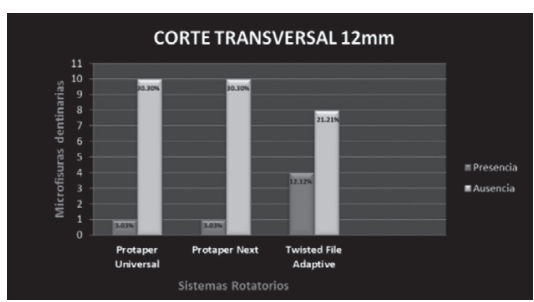

Figura $\mathrm{N}^{\circ}$ 3: Número y porcentaje de $\mathrm{Mi}$ crofisuras dentinarias a $12 \mathrm{~mm}$ después de la instrumentación con diferentes sistemas.

\section{Resultados}

El análisis estadístico mediante la prueba de Chi Cuadrado, nos demostró diferencia significativa $(p>0.05)$ entre los diferentes sistemas rotatorios (Protaper Universal, Twisted File Adaptive, Protaper Next) y la creación de microfisuras dentinales en el tercio apical $(4 \mathrm{~mm})$. Sin embargo, en el tercio medio $(8 \mathrm{~mm})$ y tercio Cervical $(12 \mathrm{~mm})$ no se encontró diferencia significativa.

En la figura $n^{\circ} 3$, se observa que el sistema Protaper Universal presenta 30.3\% frente al 6.06\% del sistema Twisted File Adaptive ( $>0.05$ ); y, el 9.09\% del sistema Protaper Next en la generación de microfisuras dentinales en la preparación químico mecánica.

En la figura $\mathrm{n}^{\circ} 4$, se observa que el sistema Protaper Universal presenta 6.6\%\% frente al $12.12 \%$ del sistema Twisted File Adaptive; y, el 9.09\% del sistema Protaper Next en la generación de microfisuras dentinales en la preparación químico mecánica.

En la Figura $n^{\circ} 5$, se observa que el sistema Protaper Universal presenta $3.03 \%$ al igual que el sistema Twisted File Adaptive, mientras que el sistema Protaper Next presenta $12.12 \%$; en la 
generación de microfisuras dentinales en la preparación químico mecánica.

\section{Discusión}

Los 3 sistemas utilizados en el presente estudio causaron microfisuras tal como reportan Karatas et al, Liu et al, DeDeus et al. ${ }^{10-12}$

La mayor cantidad de microfisuras en el tercio apical fueron producidas por el sistema Protaper Universal, Liu et al sugieren que ésto se puede deber al taper mayor que presenta el sistema que es de 0.8 en comparación al del Protaper Next (0.6) y TF Adaptive (0.6), lo cual concuerda por lo hallado por Karatas et al. ${ }^{13}$

Además el sistema TF Adaptive, según el fabricante trabaja con el menor stress posible, ya que al encontrar stress en la conformación del conducto, cambiará su movimiento en sentido contrario, reduciendo el stress en la conformación por lo que justificaría la poca presencia de microfisuras en comparacion al Sistema Protaper Universal. Burklein et al nos dicen que el sistema Protaper Next presenta una sección transversal de diseño rectangular por lo que ésto también influiría en la menor presencia de mcirofisuras, coincidiendo con los resultados del presente estudio.

De acuerdo a muchos estudios (Liu etal, Hin et al, Yoldas et al, Karatas et al), los dientes fueron examinados previamente en el estéreo microscopio, y se utilizaron controles negativos.

Coincidiendo con Karatas et al, Capar et al, Liu et las microfisuras fueron observadas después de seccionar los dientes a diferentes distancias desde apical; sin embargo De-Deus et al ${ }^{14-15}$ nos dicen que el método adecuado para la observación sería a través de una micro tomografía sin realizar ningún corte transversal a los dientes evitando así los posibles falsos positivos al cortar los dientes.

\section{Conclusiones}

1. Se puede concluir que los sistemas PROTAPER NEXT y TWISTED FILE ADAPTIVE tienden a generar menor cantidad de microfisuras dentinarias en comparación al sistema PROTAPER UNIVERSAL en el tercio apical.

\section{Referencias Bibliográficas}

1. Tesis I, Rosenberg E, Faivishevsky $\mathrm{V}$, et al. Prevalence and associated periodontal status of teeth with root perforation: a retrospective study of 2,002 patients' medical records. J Endod 2010;36:797-800

2. Aydin B, Kose T, Caliskan MK. Effectiveness of HERO 642 versus Hedstrom files for removing gutta-percha fillings in curved root canals: an ex vivo study. Int Endod J 2009; 42:1050-6.

3. Cuje J, Bargholz C, Hulsmann M. The outcome of retained instrument removal in a specialist practice. Int Endod J 2010; 43:545-54.

4. Capar ID, Arslan H, Akcay M, et al. Effects of ProTaper Universal, ProTaper Next, and HyFlex instruments on crack formation in dentin. J Endod 2014; 40:1482-4.

5. Wilcox LR, Roskelley C, Sutton T. The relationship of root canal enlargement to finger-spreader induced vertical root fracture. J Endod 1997; 23:533-4.

6. Tamse A, Fuss Z, Lustig J, et al. An evaluation of endodontically treated vertically fractured teeth. J Endod 1999; 25:506-8.

7. Adorno CG, Yoshioka T, Suda H. The effect of working length and root canal preparation technique on crack development in the apical root canal wall. Int Endod J 2010; 43:321-7.

8. Burklein S, Tsotsis P, Schafer E. Incidence of dentinal defects after root canal preparation: reciprocat- ing versus rotary instrumentation. J Endod 2013; 39:501-4.

9. Bier CA, Shemesh H, TanomaruFilho M, et al. The ability of different nickel-titanium rotary instruments to induce dentinal damage during canal preparation. J Endod 2009; 35:236-8. 15. Liu R, Kaiwar A, Shemesh $\mathrm{H}$, et al. Incidence of apical root cracks and apical dentinal detachments after canal preparation with hand and rotary files at different instrumentation lengths. J Endod 2013; 39:129_ 32 .

10. Liu R, Kaiwar A, Shemesh H, et al. Incidence of apical root cracks and apical dentinal detachments after canal preparation with hand and rotary files at different instrumentation lengths. J Endod 2013; 39:129-32.

11. Shemesh H, Bier CA, Wu MK, et al. The effects of canal preparation and filling on the incidence of dentinal defects. Int Endod J 2009; 42:208-13.

12. Liu R, Hou BX, Wesselink PR, et al. The incidence of root microcracks caused by 3 different singlefile systems versus the ProTaper system. J Endod 2013; 39:1054-6.

13. Karatas, E. Dentinal Crack Formation during Root Canal Preparations by the Twisted File Adaptive, ProTaper Next, ProTaper Universal, and WaveOne Instruments. J Endod 2015;-:1-4

14. De-Deus G. Lack of Causal Relationship between Dentinal Microcracks and Root Canal Preparation with Reciprocation Systems. J En$\operatorname{dod} 2014$

15. De-Deus G. Micro-computed Tomographic Assessment on the Effect of ProTaper Next and Twisted File Adaptive Systems on Dentinal Cracks. JOE 2015 